\title{
Multimodality molecular imaging: Paving the way for personalized medicine
}

Type of article: Conference abstract

\author{
Habib Zaidi ${ }^{1,2,3,4}$ \\ 1Division of Nuclear Medicine and Molecular Imaging, Geneva University Hospital, \\ Switzerland \\ 2Department of Nuclear Medicine and Molecular Imaging, University of Groningen, The \\ Netherlands \\ 3Department of Nuclear Medicine, University of Southern Denmark, Odense, Denmark \\ 4Institute for Advanced Studies, University of Cergy-Pontoise, Cergy, France \\ Email: habib.zaidi@hcuge.ch \\ Web: http://pinlab.hcuge.ch/
}

\begin{abstract}
Early diagnosis and therapy increasingly operate at the cellular, molecular or even at the genetic level. As diagnostic techniques transition from the systems to the molecular level, the role of multimodality molecular imaging becomes increasingly important. Positron emission tomography (PET), x-ray CT and MRI are powerful techniques for in vivo imaging. The inability of PET to provide anatomical information is a major limitation of standalone PET systems. Combining PET and CT proved to be clinically relevant and successfully reduced this limitation by providing the anatomical information required for localization of metabolic abnormalities. However, this technology still lacks the excellent soft-tissue contrast provided by MRI. Standalone MRI systems reveal structure and function, but cannot provide insight into the physiology and/or the pathology at the molecular level. The combination of PET and MRI, enabling truly simultaneous acquisition, bridges the gap between molecular and systems diagnosis. MRI and PET offer richly complementary functionality and sensitivity; fusion into a combined system offering simultaneous acquisition will capitalize the strengths of each, providing a hybrid technology that is greatly superior to the sum of its parts.

This talk also reflects the tremendous increase in interest in quantitative molecular imaging using PET as both clinical and research imaging modality in the past decade. It offers a brief overview of the entire range of quantitative PET imaging from basic principles to various steps required for obtaining quantitatively accurate data from dedicated standalone PET and combined PET/CT and PET/MR systems including algorithms used to correct for physical degrading factors and to quantify tracer uptake and volume for radiation therapy treatment planning. Future opportunities and the challenges facing the adoption of multimodality imaging technologies and their role in biomedical research will also be addressed.
\end{abstract}

Keywords: Medical Imaging, Multimodality imaging, Personalized medicine. 


\section{Declaration of conflicts}

This article is a keynote presented at the International Conference on Health Sciences and Medical Technologies 2017 ICHSMT'17.

\section{Authors' biography}

Professor Habib Zaidi is head of the PET Instrumentation \& Neuroimaging Laboratory at Geneva University Hospital and faculty member at the medical school of Geneva University. He is also a Professor of Medical Physics at the University of Groningen (Netherlands), Adjunct Professor of Medical Physics and Molecular Imaging at the University of Southern Denmark and visiting Professor at IAS/University Cergy-Pontoise (France). He is actively involved in developing imaging solutions for cutting-edge interdisciplinary biomedical research and clinical diagnosis in addition to lecturing undergraduate and postgraduate courses on medical physics and medical imaging. His research is supported by the Swiss National Foundation, private foundations and industry (Total 4.65 M US\$) and centres on hybrid imaging (PET/CT and PET/MRI). He was guest editor for 10 special issues of peer-reviewed journals and is a member of the editorial board of leading journals in medical imaging. He has been elected as senior member of the IEEE and liaison representative of the International Organization for Medical Physics (IOMP) to the World Health Organization (WHO) in addition to being affiliated to several International medical physics and nuclear medicine organisations. He is developer of physics web-based instructional modules for the RSNA and Editor of IPEM's Nuclear Medicine web-based instructional modules. $\mathrm{He}$ is also $\mathrm{He}$ is involved in the evaluation of research proposals for European and International granting organisations and participates in the organisation of International symposia and top conferences as member of scientific committees. His academic accomplishments in the area of quantitative PET imaging have been well recognized by his peers and by the medical imaging community at large since he is a recipient of many awards and distinctions among which the prestigious 2003 Young Investigator Medical Imaging Science Award given by the Nuclear Medical and Imaging Sciences Technical Committee of the IEEE, the 2004 Mark Tetalman Memorial Award given by the Society of Nuclear Medicine, the 2007 Young Scientist Prize in Biological Physics given by the International Union of Pure and Applied Physics (IUPAP), the prestigious (100’000\$) 2010 kuwait Prize of Applied sciences (known as the Middle Eastern Nobel Prize) given by the Kuwait Foundation for the Advancement of Sciences (KFAS) for "outstanding accomplishments in Biomedical technology", the 2013 John S. Laughlin Young Scientist Award given by the American Association of Physicists in Medicine (AAPM), the 2013 Vikram Sarabhai Oration Award given by the Society of Nuclear Medicine, India (SNMI) and the 2015 Sir Godfrey Hounsfield Award given by the British Institute of Radiology (BIR). Prof. Zaidi has been an invited speaker of over 130 keynote lectures and talks at an International level, has authored over 230 peerreviewed journal articles in prominent journals and is the editor of three textbooks on Therapeutic Applications of Monte Carlo Calculations in Nuclear Medicine, Quantitative Analysis in Nuclear Medicine Imaging and Molecular Imaging of Small Animals. 
Medical Technologies Journal, Volume: 1, Issue: 3, July -September 2017, Pages: 44-46. DOI: https://doi.org/10.26415/2572-004X-vol1iss3p44-46

\section{References}

Zaidi $\mathbf{H}$ and Becker $\mathrm{M}$ "The promise of hybrid PET-MRI: Technical advances and clinical applications" IEEE Signal Process Mag Vol. 33, No. 3, pp 67-85 (2016).

Mehranian A, Arabi H and Zaidi H "Vision 20/20: Magnetic resonance imagingguided attenuation correction in PET/MRI: Challenges, solutions and opportunities" Med Phys Vol. 43, No. 3, pp 1130-1155 (2016).

Zaidi $\mathbf{H}$ et al "PET performance of the Ingenuity TF PET-MR: a whole body PETMRI system" Phys Med Biol Vol. 56, No. 10, pp 3091-3106 (2011). 